\title{
BOUNDARY BEHAVIOR OF CONFORMAL AND QUASICONFORMAL MAPPINGS
}

\author{
DOV AHARONOV and URI SREBRO
}

Dedicated to our teacher Professor Elisha Netanyahu

\section{Introduction}

1.1. Let $b$ be a boundary point of a simply connected domain $D$ in $\hat{\mathbf{C}}=\hat{\mathbf{C}} \cup\{\infty\}$ and suppose that $D$ is locally $n$-connected at $b, n \geqq 1$. This means that $n$ is the smallest integer having the property that $b$ has arbitrarily small neighborhoods $V$ such that $D \cap V$ has exactly $n$ connected components. An example of such a domain is the complement in $\hat{\mathbf{C}}$ of, what we call, a straight $n$-star $S$, which is (by our definition) a union of $n$ finite line segments $I_{j}, 1 \leqq j \leqq n$, possibly of different length, having a common end point at 0 and being evenly spaced. There exists a conformal map $f$ of $D$ onto $\hat{\mathbf{C}} \backslash S$ such that $f(z) \rightarrow 0$ as $z \rightarrow b$. A question then arises: How does $f$ grow near 0 ?

This problem is related to distortion problems near the boundary for conformal mappings in $B=\{z \in \mathbf{C}:|z|<1\}$ and for quasiconformal mappings in $B^{n}=$ $\left\{x \in R^{n}:|x|<1\right\}$ and to growth problems near isolated essential singularities of meromorphic mappings.

We studied one of these problems in [2] showing how the solution to the problem implies simply and naturally the Denjoy-Carleman-Ahlfors theorem. The basic tools in [2] were inequalities for the logarithmic capacity. We now use modulus of path families as a tool. This makes our new proof of Denjoy-Carleman-Ahlfors theorem look similar in someway to Macintyre's [9].

The next chapter contains basic definitions and results which are used in the following chapters. Chapters 3 and 4 refer to mappings in the complex plane $\mathbf{C}$ or on the Riemann sphere $\hat{\mathbf{C}}=\mathbf{C} \cup\{\infty\}$. The rest of the paper deals with mappings in $R^{n}, n \geqq 2$. All conformal or quasiconformal mappings which are considered here are assumed to be injective.

Acknowledgement. We wish to thank Professors M. Esseń, F. D. Lesley, A. Marden and B. Palka for helpful conversations. The first author wishes to thank the Technion's foundation for promotion of research for a research grant. The second author wishes to thank Professor M. Heins and University of Maryland in College Park for a research grant. 


\section{Notation and preliminaries}

2.1. For $a=\left(a_{1}, \ldots, a_{n}\right) \in R^{n}$ and $r \in(0, \infty)$ we let $|a|=\left(\sum_{i=1}^{n} a_{i}^{2}\right)^{1 / 2}, B^{n}(a, r)=$ $\left\{x \in R^{n}:|a-x|<r\right\}, B^{n}(r)=B^{n}(0, r), B^{n}=B^{n}(1) B=B^{2}, R_{+}^{n}=\left\{x \in R^{n}: x_{n}>0\right\} \quad$ and $B_{+}^{n}=B^{n} \cap R_{+}^{n}$. For $f: D \rightarrow \bar{R}^{n}, D \subset R^{n}$, denote $M_{f}(r)=\sup \{|f(x)|: x \in D,|x|=r\}$. The cluster set of a function $f: D \rightarrow \bar{R}^{n}$ at a boundary point $b \in \partial D$ is denoted by $C(f, b)$. The cluster set of $f$ on a set $E \subset \partial D$ is defined by $C(f, E)=\bigcup_{b \in E} C(f, b)$.

The modulus of a family $\Gamma$ of paths $\gamma:[0,1] \rightarrow \bar{R}^{n}$ is defined by

$$
M(\Gamma)=\inf \int_{R^{n}} \varrho^{n} d m
$$

where the infimum is taken over all non-negative Borel functions $\varrho: R^{n} \rightarrow R^{1}$ which satisfy the condition

$$
\int_{\gamma} \varrho|d x| \geqq 1
$$

for all locally rectifiable paths $\gamma \in \Gamma$.

For an open set $D$ in $\bar{R}^{n}$ and compact sets $E$ and $F$ in $D$ we let $\Gamma(E, F, D)$ denote the family of paths joining $E$ and $F$ in $D$, and we let $\Gamma(E, D)=\Gamma(E, \partial D, D)$.

2.2. The outer dilation of a quasiconformal mapping $f: D \rightarrow \bar{R}^{n}, D \subset R^{n}, n \geqq 2$, is defined by

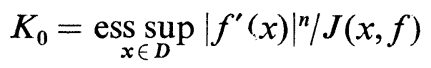

where $f^{\prime}(x)$ is the formal derivative of $f,\left|f^{\prime}(x)\right|$ is the norm of the operator $f^{\prime}(x): R^{n} \rightarrow R^{n}$ and $J(x, f)=\operatorname{det} f^{\prime}(x)$.

Note that $f$ is conformal if and only if $K_{0}=1$.

2.3. The invariance of the modulus. Let $\Gamma$ be a path family in a domain $D \subset \bar{R}^{n}$ and $f: D \rightarrow \bar{R}^{n}$ a quasiconformal mapping with outer dilation $K_{0}$. Then, see [11],

$$
M(\Gamma) \leqq K_{0} M(f(\Gamma)) .
$$

Equality holds if and only if $f$ is conformal.

2.4. A symmetry principle; cf. [3]. Let $\varphi: R^{n} \rightarrow R^{n}$ denote the reflection in $\partial R_{+}^{n}, E$ a continuum in $\bar{B}_{+}^{n}, \Gamma=\Gamma\left(E, \partial B^{n}, B_{+}^{n}\right)$ and $\Gamma^{\prime}=\Gamma\left(E \cup \varphi(E), B^{n}\right)$. Then

$$
M(\Gamma)=M(\varphi(\Gamma))=\frac{1}{2} M\left(\Gamma^{\prime}\right) .
$$

2.5. Lemma. Let $\Gamma_{1}$ and $\Gamma_{2}$ be path families in $\bar{R}^{n}$ such that $\Gamma_{2}$ is minorized by $\Gamma_{1}, \Gamma_{1}<\Gamma_{2}$, meaning that each $\gamma_{2} \in \Gamma_{2}$ has a subpath $\gamma_{1} \in \Gamma_{1}$. Then $M\left(\Gamma_{2}\right) \leqq M\left(\Gamma_{1}\right)$.

Proof follows from the definition. Cf. [11, 6.4].

2.6. Superadditivity. Let $0<r<R<\infty$ and let $\Gamma_{1}, \ldots, \Gamma_{m}$ be path families which, respectively, lie in disjoint Borel sets $D_{1}, \ldots, D_{m}$ in $R^{n}$, such that 
$\Gamma=\bigcup_{i=1}^{m} \Gamma_{i} \subset \Gamma\left(\bar{B}^{n}(r), B^{n}(R)\right)$. Then

$$
M(\Gamma) \leqq \omega_{n-1}\left(\log \frac{R}{r}\right)^{1-n} .
$$

See [11, 6.7 and 7.5].

2.7. Lemma. Let $E$ be a continuum in $B^{n}$ which contains the point 0 and meets $\partial B^{n}(r), 0<r<1$, and let $\Gamma=\Gamma\left(E, B^{n}\right)$. Then

$$
M(\Gamma) \geqq \omega_{n-1}\left(\log \frac{\lambda_{n}}{r}\right)^{1-n}
$$

where $\lambda_{n}>0$ depends only on $n$ and $\lambda_{2}=4$. Cf. [7, Ch. II] and [4, p. 235].

2.8. Lemma. Let $0<r<1,0<\alpha \leqq 2 \pi, G=\{z \in \mathbf{C}:|z|<1,0<\arg z<\alpha\}$ and $E$ a continuum in $\bar{G}$ which contains the point 0 and meets $\partial B(r)$. Let $\Gamma=$ $\Gamma(E, \partial B \cap \bar{G}, G)$. Then

$$
M(\Gamma) \geqq \pi\left(\log 4 r^{-\pi / \alpha}\right)^{-1} .
$$

Proof. Let $\psi(z)=z^{\pi / \alpha}, \varphi(z)=\bar{z}, E^{\prime}=\psi(E) \cup \varphi(\psi(E))$ and $\Gamma^{\prime}=\Gamma(E, B) . \quad$ Then $M(\Gamma)=M\left(\Gamma^{\prime}\right) / 2$ by 2.3 and 2.4. The result follows now by 2.7 .

2.9. Lemma. Let $d>0, R>0$ and $a \in \partial B^{n}, n>2$, such that $\bar{B}^{n}(a, d) \subset B=$ $B^{n}\left(\sqrt{1+R^{2}} a, R\right)$. Let $E$ be a continuum in $\bar{B}^{n} \cap B$ which contains the point 0 and meets $\partial B^{n}(a, d)$ and let $\Gamma=\Gamma\left(E, \partial B \cap B^{n}, B \cap B^{n}\right)$. Then

$$
M(\Gamma) \geqq \frac{\omega_{n-1}}{2} \cdot\left(\log \frac{\lambda_{n} R}{d}\right)^{1-n}
$$

where $\lambda_{n}$ is the constant mentioned in 2.7 .

Proof. Let $\Gamma_{0}=\Gamma(E, B)$. Since $\partial B$ is perpendicular to $\partial B^{n}$ it follows by 2.4 that $M(\Gamma)=M\left(\Gamma_{0}\right) / 2$. Let $\varphi$ be a Möbius transformation such that $\varphi(B)=B^{n}$ and $\varphi(a)=0$. Then $\varphi(E)$ is a continuum in $B^{n}$ which contains 0 and meets $\partial B^{n}\left(d^{\prime}\right)$ for some $d^{\prime}>d$, and $M\left(\Gamma_{0}\right)=M\left(\varphi\left(\Gamma_{0}\right)\right)$. This together with 2.7 gives the desired inequality

2.10. Lemma. Let $n>1$ and let $\alpha_{1}, \ldots, \alpha_{m}>0$. Then

$$
m^{n}\left(\sum_{i=1}^{m} \alpha_{i}\right)^{1-n} \leqq \sum_{i=1}^{m} \alpha_{i}^{1-n}
$$

Proof. By Hölder's inequality

$$
m^{n}=\left(\sum_{i=1}^{m} \alpha_{i}^{(n-1) / n} \alpha_{i}^{(1-n) / n}\right)^{n} \leqq\left(\sum_{i=1}^{m} \alpha_{i}\right)^{n-1} \sum_{i=1}^{m} \alpha_{i}^{1-n},
$$

and the result follows. 


\section{Mapping and distortion theorems}

3.1. Theorem. Let $D$ be a simply connected domain in $\hat{\mathbf{C}}$ with $\infty \in D, 0 \in \partial D$ and $\partial D \backslash\{0\} \neq \emptyset$, which is locally m-connected at $0 \in \partial D, m \geqq 1$. Then:

(i) There exists a conformal mapping $g$ of $D$ onto the complement of a straight $m$-star $S$ such that $g(z) \rightarrow 0$ as $z \rightarrow 0$ and having the normalizations $g(\infty)=\infty$ and $g(z) / z \rightarrow 1$ as $z \rightarrow \infty$.

(ii) Let $g$ be as in (i). For $r>0$ sufficiently small $D \cap B(r)$ has $m$ connected components $D_{j}(r)$, having the property $0 \in \partial D_{j}(r), 1 \leqq j \leqq m$. Let $M_{j}(r)=\sup \{|g(z)|$ : $\left.z \in D_{j}(r)\right\}$. Then

$$
\prod_{j=1}^{m} M_{j}(r) \leqq 16 r^{m}
$$

The constant 16 is best possible.

3.2. Proof. (i) Let $\varphi$ be a conformal map of $D$ onto $\hat{\mathbf{C}} \backslash \bar{B}$ with $g(\infty)=\infty$. Then $C(g, 0)$ consists of distinct points $a_{j} \in \partial B, 1 \leqq j \leqq m$. The function $h(z)=$ $z^{-1} \prod_{j=1}^{m}\left(z-a_{j}\right)^{2 / m}$ maps $\hat{\mathbf{C}} \backslash \bar{B}$ onto the complement of a straight $m$-star with $h(\infty)=\infty$ and $h\left(a_{j}\right)=0,1 \leqq j \leqq m$. Therefore for a suitable constant $A, g(z)=$ $A h(\varphi(z))$ will have the desired properties.

(ii) By extending the arms of $S$ to $\infty, \mathrm{C}$ is divided into $m$ congruent sectors $D_{j}^{\prime}, 1 \leqq j \leqq m$. Let $D_{j}=g^{-1}\left(D_{j}^{\prime}\right)$. It is clear that for all sufficiently small $r>0$, $D \cap B(r)$ will have exactly $m$ connected components $D_{j}(r), 1 \leqq j \leqq m$, having the property that $D_{j}(r) \subset D_{j}$ and $0 \in \partial D_{j}(r)$. Choose $R>0$ large enough so that $\widehat{\mathbf{C}} \backslash B(R) \subset D$. Let $R^{\prime}=M_{g}(R), \Gamma_{j} ;=\Gamma\left(\bar{D}_{j}(r), \partial B(R), D_{j}\right) \quad$ and $\quad \Gamma_{j}^{\prime} ;=\Gamma \overline{\left(g\left(D_{j}(r)\right.\right.}$, $\left.\partial B\left(R^{\prime}\right), D_{j}^{\prime}\right)$. Then

$$
\sum_{j=1}^{m} \frac{\pi}{\log 4\left(R^{\prime} / M_{j}\right)^{m / 2}} \leqq \sum_{j=1}^{m} M\left(\Gamma_{j}^{\prime}\right) \leqq \sum_{j=1}^{m} M\left(g\left(\Gamma_{j}\right)\right)=\sum_{j=1}^{m} M\left(\Gamma_{j}\right) \leqq \frac{2 \pi}{\log (R / r)} .
$$

The first inequality follows from Lemma 2.7 , the second from 2.5 , the last from 2.6 and the equality from 2.3. This string of inequalities together with Lemma 2.10 implies

$$
\frac{1}{\log R / r} \geqq \frac{1}{m} \sum_{j=1}^{m}\left(\log 16^{1 / m} R^{\prime} / M_{j}\right)^{-1} \geqq m\left(\sum_{j=1}^{m} \log 16^{1 / m} R^{\prime} / M_{j}\right)^{-1} .
$$

Consequently, $\prod_{j=1}^{m} M_{j} \leqq 16\left(r R^{\prime} \mid R\right)^{m}$. But $R^{\prime} / R \rightarrow 1$ as $R \rightarrow \infty$ due to the normalizations of $g$, thus $\prod_{j=1}^{m} M_{j} \leqq 16 r^{n}$.

We now show that the constant 16 cannot be replaced by a smaller constant. Consider first the case $m=1$. Fix $a \in(1, \infty)$ and $\varepsilon \in(0, \pi)$ and let $f_{\varepsilon}$ denote the mapping of the complement in $\hat{\mathbf{C}}$ of the set

$$
S_{\varepsilon, a}=\{z:|z|=1, \varepsilon \leqq \arg z \leqq 2 \pi\} \cup[1, a] \cup\left\{\varrho e^{i \varepsilon}: 0 \leqq \varrho \leqq 1\right\}
$$

onto a straight 1 -star $S=\left[0, a^{\prime}\right]$ such that $f_{\varepsilon}(z) \rightarrow 0$ as $z \rightarrow 0$ and normalized: 
$f_{\varepsilon}(\infty)=\infty$ and $f_{\varepsilon}(z) / z \rightarrow 1$ as $z \rightarrow \infty$. Here $a^{\prime}>1$ will depend on $\varepsilon$ and $a$. Such a mapping exists by the first part of the theorem. By Carathéodory's convergence theorem $f(z)=\lim _{+\varepsilon \rightarrow 0} f_{\varepsilon}(z)$ exists and defines a conformal mapping of the kernel $D=\widehat{\mathbf{C}} \backslash B \backslash[1, a]$ with respect to $\infty$ of the domains $D_{\varepsilon}$ of $f_{\varepsilon}, 0<\varepsilon<\pi$, onto the slit domain $\hat{\mathbf{C}} \backslash\left[0, a^{\prime \prime}\right]$ for some $a^{\prime \prime}>1$, Since each $f_{\varepsilon}$ can be continued analytically across the slit $[1, a]$ the convergence is uniform with respect to the spheric metric in $\hat{\mathbf{C}} \backslash B(r) \backslash[1, a]$ for any $r>1$. It is also easy to see that $\operatorname{diam} f_{\varepsilon}(B) \rightarrow 0$ as $\varepsilon \rightarrow 0$, and thus $C(f, 1)=\{0, M\}$ where $0<M=\lim _{+r \rightarrow 1} \lim _{\varepsilon \rightarrow 0} M\left(r, f_{\varepsilon}\right)=$ $\lim _{+r \rightarrow 1} M(r, f)$. By computations one has $M=(16(a+(1 / a))-32) /(a+(1 / a)+2) \rightarrow 16$ as $a \rightarrow \infty$. This proves the sharpness of the inequality in (ii) in the case $m=1$.

For $m>1$ choose a branch $g_{\varepsilon}$ of $f_{\varepsilon}\left(z^{m}\right)^{1 / m}$ and get $M_{j} \approx 16^{1 / m}$ as $\varepsilon \rightarrow 0, r \rightarrow 1$ and $a \rightarrow \infty$ for all $1 \leqq j \leqq m$. This proves that the constant 16 in (ii) cannot be replaced by a smaller constant.

3.3. Remark. In the example given in $3.2 \prod_{j=1}^{m} M_{j}(r) \approx 16 r^{m}$ for one value of $r$, namely $r=1$. We do not know whether 16 is the smallest possible constant in the following inequality, which follows from Theorem 3.1. (ii)

$$
\limsup _{r \rightarrow 0} r^{-m} \prod_{j=1}^{m} M_{j}(r) \leqq 16 .
$$

We now establish a local version of Theorem 3.1.

3.4. Theorem. Let $D$ be a domain in $\hat{C}$ which is locally m-connected at $0 \in \partial D, m \geqq 1$. Suppose that the connected component $E$ of $\partial D$ which contains 0 is not a point and that $E \cap \overline{\partial D \backslash E}=\emptyset$. For $r>0$ sufficiently small $D \cap B(R)$ has $m$ simply connected components $D_{j}(r), 1 \leqq j \leqq m$, with $0 \in \overline{D_{j}(r)}$.

Let $f$ be a conformal mapping of $D$ into the complement of a straight m-star $S$ such that $C(f, E) \subset S$ and $g(z) \rightarrow 0$ as $z \rightarrow 0$. Then

$$
\limsup _{r \rightarrow 0} r^{-m} \prod_{j=1}^{m} M_{j}(r)<\infty
$$

where $M_{j}(r)=\sup _{z \in D_{j}(r)}|f(z)|$.

Proof. Let $F$ denote the connected component of $\hat{\mathbf{C}} \backslash D$ which contains $E$. Since $S$ is compact in $\mathbf{C}$ and $C(f, E)=S, F$ has a simply connected neighborhood $U$ such that $U \cap \partial D=E$ and such that $f(U) \subset B\left(R^{\prime}\right)$ for some $R^{\prime}>0$. Extend each arm of $S$ until it first meets $f(\partial U)$. This divides $f(U \cap D)$ into $m$ domains $D_{j}^{\prime}$. We may assume that the domains $D_{j}^{\prime}$ lie, respectively, in the sectors $\Delta_{j}$ which are obtained from $\mathbf{C}$, when the arms of $S$ are extended to $\infty$. If this is not the case, delete from $U$ the sets $f^{-1}\left(D_{j} \cap \Delta_{i}\right)$ for all $i \neq j$ and replace $U$ by an appropriate subset. Choose $R>0$ such that $B(R) \subset U$ and such that $D \cap B(r)$ has $m$ connected components $D_{j}(r) 1 \leqq j \leqq m$ with $0 \in \bar{D}_{j}(r)$ and $D_{j}(r) \subset D_{j}=f^{-1}\left(D_{j}^{\prime}\right)$, $1 \leqq j \leqq m$, whenever $0<r \leqq R$. For $r \in(0, R)$ and $1 \leqq j \leqq m$ let $\Gamma_{j}=\Gamma\left(\overline{D_{j}(r)}, \partial U, D_{j}\right)$ 
and $\Gamma_{j}^{\prime}=\Gamma\left(\overline{f D_{j}(r)}, \partial B\left(R^{\prime}\right), \Delta_{j}\right)$. Then

$$
M\left(\Gamma_{j}^{\prime}\right) \leqq M\left(f\left(\Gamma_{j}\right)\right)=M\left(\Gamma_{j}\right)
$$

by 2.5 and 2.3 . Next, 2.6 and 2.7 imply

and

$$
\sum_{j=1}^{m} M\left(\Gamma_{j}\right) \leqq \frac{2 \pi}{\log (R / r)}
$$

Hence

$$
\sum_{j=1}^{m} \frac{\pi}{\log 4\left(R^{\prime} / M_{j}\right)^{m / 2}} \leqq \sum_{j=1}^{m} M\left(\Gamma_{j}^{\prime}\right)
$$

$$
\frac{1}{m} \sum_{j=1}^{m}\left(\log 16^{1 / m} R^{\prime} / M_{j}\right)^{-1} \leqq(\log R / r)^{-1}
$$

and by 2.10 it follows that

$$
\prod_{j=1}^{m} M_{j} \leqq 16\left(R^{\prime} / R\right)^{m} r^{m}
$$

which implies the assertion of the theorem.

\section{Applications}

We now show how modified versions of the Denjoy-Carleman-Ahlfors theorem follow directly from Theorem 3.1 and the classical Phragmén Lindelöf principle.

4.1. Theorem. Let $E$ be a non-degenerate continuum in $\hat{\mathbf{C}}$ such that $\infty \in E$, and such that $D=\mathbf{C} \backslash E$ is locally m-connected at $\infty$.

If $f$ is analytic in $\mathbf{C}$, bounded on $E \backslash\{\infty\}$ and unbounded in each component of $D \backslash B(r)$ for all $r>0$, then $\lim _{\inf _{r \rightarrow \infty}} r^{-m / 2} \log M_{f}(r)>0$.

Proof. With no loss of generality we may assume that $0 \notin E$. By 3.1 (i) there is a conformal map of $D$ onto the complement of a straight $n$-star $S$ such that $g(z) \rightarrow 0$ as $z \rightarrow \infty, g(0)=\infty$ and $z g(z) \rightarrow 1$ as $z \rightarrow 0$. Extend the arms of $S$ to $\infty$. This divides $\mathbf{C}$ into $m$ congruent sectors $D_{j}^{\prime}, 1 \leqq j \leqq m$. Let $D_{j}=g^{-1}\left(D_{j}^{\prime}\right), g_{j}=g \mid D_{j}$. Then $F_{j}=f \circ g_{j}^{-1}$ is bounded on $\partial D_{j}^{\prime} \backslash\{0\}$ and unbounded in $D_{j}$. Thus, by the principle of Phragmén Lindelöf there are positive constants $\alpha_{j}, 1 \leqq j \leqq m$, such that $\alpha_{j} \leqq r^{m / 2} \log M_{F_{j}}(r)$ for $1 \leqq j \leqq m$ and all sufficiently small $r>0$. For sufficiently large $r, D \backslash \bar{B}(r)$ has $m$ connected components $D_{j}(r)$ such that $\infty \in \bar{D}_{j}(r)$ and $D_{j}(r) \subset D_{j}, \quad 1 \leqq j \leqq m . \quad$ Let $\quad M_{j}(r)=\sup \left\{|g(z)|: z \in D_{j}(r)\right\} . \quad$ By Theorem 3.1(ii), $\prod_{j=1}^{m} M_{j}(r) \leqq 16 r^{-m}$ and by the maximum principle $M_{F_{j}}\left(M_{j}\right) \leqq M_{f \mid D_{j}}(r) \leqq M_{f}(r)$. Hence

$$
\prod_{j=1}^{m} \alpha_{j} \leqq \sum_{j=1}^{m} M_{j}^{m / 2} \log M_{F_{j}}\left(M_{j}\right) \leqq 4^{m} r^{-m^{2} / 2} \log ^{m} M_{f}(r) .
$$


Therefore, $r^{-m / 2} \log M_{f}(r) \geqq 1 / 4\left(\prod_{j=1}^{m} \alpha_{j}\right)^{1 / m}>0$, for all sufficiently large $r$. This completes the proof.

4.2. Corollary (Denjoy-Carleman-Ahlfors). If $f$ is entire and has $m$ distinct finite asymptotic values, then $\lim \inf r^{-m / 2} \log M_{f}(r)>0$ as $r \rightarrow \infty$.

Proof. Let $\gamma_{j}, j=1, \ldots, n$ be disjoint asymptotic arcs. Then $f$ is bounded on $E=U \gamma_{j}$ and unbounded between any two adjacent arcs. The assertion follows, now, from 4.1.

4.3. Theorem. Let the point 0 be an isolated essential singularity of a holomorphic function $f$ defined in a domain $D$. Let $E$ be a non-degenerate continuum in $\mathbf{C}$ such that $0 \in E \subset D \cup\{0\}$ and such that $D \backslash E$ is connected and locally m-connected at $0, m \geqq 1$. If $f$ is bounded on $E \backslash\{0\}$ and unbounded in each component of $B(r) \backslash E$ for all $r>0$ then $\lim \inf _{r \rightarrow 0} r^{m / 2} \log M_{f}(r)>0$.

Proof. By 3.1 (i) there is a conformal mapping $g$ of $\mathbf{C} \backslash E$ onto the complement of a straight $m$-star $S$ such that $g(z) \rightarrow 0$ as $z \rightarrow 0, g(\infty)=\infty$ and $g(z) / z \rightarrow 1$ as $z \rightarrow \infty$. Extend the arms of $S$ to $\infty$. This divides $\mathbf{C}$ into $m$ congruent sectors $\Delta_{j}$, $1 \leqq j \leqq m$. Let $D_{j}=g^{-1}\left(\Delta_{j}\right), D_{j}^{\prime}=g\left(D_{j}\right)$ and $g_{j}=g \mid D_{j}$. Then each $\Gamma_{j}=f \circ g_{j}^{-1}$ is bounded on $\partial \Delta_{j} \cap D_{j}^{\prime}$ and unbounded in $D_{j}^{\prime}$. It thus follows by the principle of Phragmén-Lindelöf that there are positive constants $\alpha_{j}, 1 \leqq j \leqq m$, such that $\alpha_{j} \leqq r^{m / 2} \log M_{F_{j}}(r)$ for all sufficiently small $r>0$. Since 0 is an isolated boundary point of $\partial D$ and since $D \backslash E$ is locally $m$-connected at 0 it follows that for sufficiently small $r>0, B(r) \cap D \backslash E$ has $m$ components $D_{j}(r)$ having the property that $0 \in \partial D_{j}(r)$ and $D_{j}(r) \subset D_{j}$. Let $M_{j}(r)=\sup _{z \in D_{j}(r)}|g(z)|$. Then by 3.1 $\prod_{j=1}^{m} M_{j}(r) \leqq 16 r^{m}$. By the maximum principle $M_{F_{j}}\left(M_{j}(r)\right) \leqq M_{f_{j}}(r) \leqq M_{f}(r)$. Hence

$$
\prod_{j=1}^{m} \alpha_{j} \leqq \prod_{j=1}^{m} M_{j}(r)^{m / 2} \log M_{F_{j}}\left(M_{j}(r)\right) \leqq 4^{m} r^{m^{2} / 2} \log ^{m} M_{f}(r)
$$

and so $\lim \inf _{r \rightarrow 0} r^{m / 2} \log M(r)>0$.

4.4. Corollary. Let 0 be an isolated essential singularity of a holomorphic map $f$. If $f$ has $m$ distinct finite asymptotic limits at 0 , then $\lim \inf _{r \rightarrow 0} r^{m / 2} \log M(r)>0$.

Proof. Let $\gamma_{j}, 1 \leqq j \leqq m$ be $m$ disjoint asymptotic arcs, then $E=\bigcup_{j=1}^{m} \gamma_{j}$ satisfies the conditions of Theorem 4.3., $f$ is bounded on $E$ and by Lindelöf's theorem unbounded between any two adjacent arcs. The corollary then follows by 4.3 .

4.5. Theorem. Let $f$ be analytic in $U=\{z: \operatorname{Im} z>0\}$ having $m, m>1$, distinct asymptotic limits at the point 0 . Then $\lim \inf _{r \rightarrow 0} r^{(m-1) / 4} \log M(r)>0$ where $M(r)=\sup _{|z|=r, z \in U}|f(z)|$.

Proof. Let $\gamma_{1}, \ldots, \gamma_{m}$ be the asymptotic paths associated with the asymptotic limits. We may assume that $\gamma_{1}$ and $\gamma_{m}$ start at the point $i$, that $\gamma_{2}, \ldots, \gamma_{m-1}$ 
lie between $\gamma_{1}$ and $\gamma_{m}$ and that $\left|\gamma_{i}\right| \cap\left|\gamma_{j}\right|=\{0\}$ for all pairs $\{i, j\} i \neq j$ other than $\{1, m\}$, for which the intersection consists of the end points $i$ and 0 . Let $D$ denote the bounded component of $U \backslash \bigcup_{i=1}^{m}\left|\gamma_{i}\right|$ and $D^{\prime}=\Psi(D)$ where $\Psi(z)=z^{2}$ and let $M^{\prime}(r)=\sup \left\{\mid f\left(\Psi^{-1}(z)|:| z \mid=r z \in D^{\prime}\right\}\right.$. Then $M^{\prime}\left(r^{2}\right) \leqq M(r)$ and, as a consequence of 4.1., $\lim \inf r^{m-1 / 2} \log M^{\prime}(r)>0$ as $r \rightarrow 0$. Therefore $\lim \inf f^{m-1 / 4} \log M(r)>0$.

\section{Distortion theorems for conformal and quasiconformal mappings}

We now study the growth of conformal and quasiconformal mappings $f: B^{n} \rightarrow \bar{R}^{n}$ near the boundary. Suppose that $y=\lim _{x \rightarrow a} f(x)$ for some $a \in \partial B^{n}$. The growth of $|f(x)|$ or of $|f(x)-y|$, if $y \neq \infty$, near $a$ is usually estimated in terms of $|x|$ or equivalently in terms of the distance of $x$ from $\partial B^{n}$; cf. [10] or [6], [8] and [5]. Here, the growth will be estimated in terms of the distance from the point $a$.

A particular attention is given to the case where $f$ assumes the same limit at several boundary points. We show that this imposes certain restrictions on the growth of $f$. And conversely, if a certain rather mild growth condition is imposed on $f$, then the degree of $f \mid \partial B^{n}$ must be bounded. This generalizes results by Essén [5], see also [1], and Spencer [10] in $\mathbf{C}$ and results by Miniowitz [8] in $R^{n}, n \geqq 2$.

5.1. Theorem. Let $f: B^{n} \rightarrow \bar{R}^{n}, n \geqq 2$, be a quasiconformal mapping such that $f(x) \rightarrow 0$ as $x \rightarrow a_{i}$ for $m \geqq 1$ distinct points $a_{i} \in \partial B^{n}, 1 \leqq i \leqq m$. Then there exists $r_{0}>0$ such that $m$ components, say $D_{i}^{\prime}(r), 1 \leqq i \leqq m$, of $B^{n}(r) \cap f B^{n}$ have the property that $0 \in \partial D_{i}^{\prime}(r), a_{i} \in \partial D_{i}(r)$ where $D_{i}(r)=f^{-1}\left(D_{i}^{\prime}(r)\right)$ and such that

$$
\left(\prod_{i=1}^{m} d_{i}\right)^{1 / m} \leqq c r^{\alpha}
$$

for all $r \in\left(0, r_{0}\right]$. Here $d_{i}=d_{i}(r)=\sup _{x \in D_{i}(r)}\left|x-a_{i}\right| \alpha=\left(m / 2 K_{0}\right)^{1 / n-1}$ and $c>0$ is a constant which does not depend on $r$.

Proof. Choose $R>0$ such that the balls $B_{i}=B^{n}\left(\sqrt{1+R^{2}} a_{i}, R\right), \quad 1 \leqq i \leqq m$, are disjoint and $r_{0}>0$ such that $D_{i}(r) \subset B_{i}$ for $0<r \leqq r_{0}$ and $1 \leqq i \leqq m$. Let $\Gamma_{i}^{\prime}=\Gamma\left(\overline{D_{i}^{\prime}(r)}, \partial B^{n}\left(r_{0}\right), D_{i}^{\prime}\left(r_{0}\right)\right)$. Each family $\Gamma_{i}=\Gamma\left(\overline{D_{i}(r)}, \partial B_{i}, B^{n}\right)$ is minorized by $f^{-1}\left(\Gamma_{i}^{\prime}\right)$ and thus $M\left(\Gamma_{i}\right) \leqq M\left(f^{-1}\left(\Gamma_{i}^{\prime}\right)\right), 1 \leqq i \leqq m$, by 2.5 . Now, 2.3. and 2.6. imply

$$
\sum_{i=1}^{m} M\left(f^{-1}\left(\Gamma_{i}^{\prime}\right)\right) \leqq K_{0} \sum_{i=1}^{m} M\left(\Gamma_{i}^{\prime}\right) \leqq K_{0} \omega_{n-1}\left(\log \frac{r_{0}}{r}\right)^{1-n}
$$

and on the other hand

by 2.9. Therefore

$$
\sum_{i=1}^{m} \frac{\omega_{n-1}}{2}\left(\log \frac{\lambda_{n} R}{d_{i}}\right)^{1-n} \leqq \sum_{i=1}^{m} M\left(\Gamma_{i}\right)
$$

$$
\sum_{i=1}^{m}\left(\log \frac{\lambda_{n} R}{d_{i}}\right)^{1-n} \leqq 2 K_{0}\left(\log \frac{r_{0}}{r}\right)^{1-n}
$$


Application of 2.10. with $\alpha_{i}=\log \left(\lambda_{n} R / d_{i}\right)$ yields

$$
m^{n-1}\left(\log \prod_{i=1}^{m} \frac{\lambda_{n} R}{d_{i}}\right)^{1-n} \leqq \frac{2 K_{0}}{m}\left[\log \frac{r_{0}}{r}\right)^{1-n} .
$$

Hence $\left(\prod_{i=1}^{m} d_{i}\right)^{1 / m} \leqq c r^{\alpha}$ for $r \in\left(0, r_{0}\right]$ where $c=\lambda_{n} R / r_{0}^{\alpha}$ and $\alpha=\left(m / 2 K_{0}\right)^{1 / n-1}$.

5.2. Corollary. Suppose that $f: B^{n} \rightarrow \bar{R}^{n}, n \geqq 2$, is quasiconformal and that $y=\lim _{x \rightarrow a_{i}} f(x)$ exists for some $m \geqq 1$ points $a_{i} \in \partial B^{n}, 1 \leqq i \leqq m$, and a point $y \in \bar{R}^{n}$. Let

and

$$
M(r)=\max _{1 \leqq i \leqq m} \sup _{\left|x-a_{i}\right|=r}|f(x)-y| \text { if } y \neq \infty
$$

Then

$$
M(r)=\min _{1 \leqq i \leqq m} \inf _{\left|x-a_{i}\right|=r}|f(x)| \quad \text { if } \quad y=\infty .
$$

$$
\liminf _{r \rightarrow 0} r^{-\beta} M(r)>0 \quad \text { if } \quad y \neq \infty
$$

and

(b)

$$
\limsup _{r \rightarrow 0} r^{\beta} M(r)<\infty \text { if } y=\infty,
$$

where $\beta=\left(2 K_{0} / m\right)^{1 / n-1}$.

Proof. It is enough to consider the case $y=0$, since the general case can be reduced to the case $y=0$ by composing $f$ with either a translation or a Möbius transformation which maps $\infty$ to 0 . Let $r_{0}$ be as in Theorem 5.1. Pick $r_{1}>0$ such that $M(r) \leqq r_{0}$ for $r<r_{1}$. Let $D_{i}^{\prime}(M(r))$ denote the connected component of $B^{n}(M(r)) \cap f\left(B^{n}\right)$ which contains $f\left(B^{n}\left(a_{i}, r\right) \cap B^{n}\right), \quad D_{i}(M(r))=f^{-1}\left(D_{i}^{\prime}(M(r))\right.$ and $d_{i}=\sup _{x \in D_{i}(M(r))}\left|x-a_{i}\right|, 1 \leqq i \leqq m$. Then by 5.1

$$
r \leqq \min d_{i} \leqq\left(\prod_{i=1}^{m} d_{i}\right)^{1 / m} \leqq c M(r)^{\alpha}
$$

where $\alpha=1 / \beta=\left(m / 2 K_{0}\right)^{1 / n-1}$ and $c>0$ is a constant which does not depend on $r$. This implies the assertion of the theorem.

5.3. Corollary. Let $f: \bar{B}^{n} \rightarrow \bar{R}^{n}$ be a continuous mapping which is quasiconformal in $B^{n}$ and let $m>1$ be an integer. Suppose that each point $a \in \partial B^{n}$ has a path $\gamma_{a}:[0,1) \rightarrow B^{n}$ such that $\gamma_{a}(t) \rightarrow a$ as $t \rightarrow 1^{-}$and such that for some $\gamma>$ $\left(2 K_{0} / m\right)^{1 / n-1}$

$$
\limsup _{\substack{x \rightarrow a \\ x \in\left|\gamma_{a}\right|}} \frac{|f(x)-f(a)|}{|x-a|^{\gamma}}<\infty \text { if } f(a) \neq \infty
$$

and

$$
\liminf _{\substack{x \rightarrow a \\ x \in\left|\gamma_{a}\right|}}|x-a|^{\gamma}|f(x)|>0 \text { if } f(z)=\infty \text {. }
$$

Then at most $m-1$ distinct points on $\partial B^{n}$ can have the same image $y$.

Proof. Suppose that $m$ distinct points $a_{i} \in \partial B^{n}, 1 \leqq i \leqq m$, have the same image $y$. Suppose first that $y \neq \infty$. Let $r_{0}, D_{i}(r)$ and $d_{i}$ be as in Theorem 5.1 
and suppose that $r_{0}$ is sufficiently small so that $\left|\gamma_{a_{i}}\right| \cap \partial D_{i}\left(r_{0}\right) \neq \emptyset$ for $1 \leqq i \leqq m$. For each $r \in\left(0, r_{0}\right]$ choose an index $i$ such that $d_{i}=\min _{1 \leqq j \leqq m} d_{j}$ and a point $x_{i} \in\left|\gamma_{a_{i}}\right| \cap \partial D_{i}(r)$. Then by 5.1 ,

$$
\left|x_{i}-a_{i}\right| \leqq d_{i} \leqq\left(\prod_{j=1}^{m} d_{j}\right)^{1 / m} \leqq c r^{\alpha}=c\left|f\left(x_{i}\right)-f\left(a_{i}\right)\right|^{\alpha},
$$

for some constant $c>0$ where $1 / \alpha=\left(2 K_{0} / m\right)^{1 / n-1}>\gamma$. It thus follows that $\mid f\left(x_{i}\right)-$ $f\left(a_{i}\right)|/| x_{i}-\left.a_{i}\right|^{\gamma} \rightarrow \infty$ as $r \rightarrow 0$ contradicting (a). Similarly, the case $y=\infty$ contradicts (b).

5.4. Remark. The last corollary is void in the event that $f$ is meromorphic $\left(n=2, K_{0}=1\right)$, unless $m \geqq 3$. A particular case of 5.3 when $m=3\left(n=2, K_{0}=1\right)$ was conjectured by Piranian and proved for $m \geqq 3$ by Essén [5] and by Aharonov [1].

\section{References}

[1] AhaRonov, D.: (to appear).

[2] Aharonov, D., and U. Srebro: A short proof of the Denjoy conjecture. - Bull. Amer. Math. Soc. (N. S.) 4, 1981, 325-328.

[3] AhLrors, L. V.: Lectures on quasiconformal mappings. - D. Van Nostrand Company, Inc., Princeton, New Jersey-Toronto-New York-London, 1966.

[4] Caraman, P.: $n$-Dimensional quasiconformal mappings. - Abacus Press, Tunbridge Wells, Kent, England, 1974.

[5] Essén, M.: Boundary behavior of univalent functions satisfying a Hölder condition. - Proc. Amer. Math. Soc. 83, 1981, 83-84.

[6] Hayman, W. K.: Multivalent functions. - Cambridge University Press, London, 1958.

[7] Lehto, O., and K. I. VIRTANeN: Quasiconformal mappings in the plane. - Springer-Verlag, Berlin-Heidelberg-New York, 1973.

[8] MinIowirz, R.: Spherically mean $p$-valent quasiregular mappings. - Israel J. Math. 38, 1981, 199-208.

[9] Macintyre, A. J.: On the asymptotic paths of integral functions of finite order. - J. London Math. Soc. (2) $10,1935,34-39$.

[10] Spencer, D. C.: On finitely mean valent functions. II. - Trans. Amer. Math. Soc. 48, 1940 , $418-435$.

[11] VÄISÄL̈̈, J.: Lectures on $n$-dimensional quasiconformal mappings - Lecture Notes in Mathematics 229, Springer-Verlag, Berlin-Heidelberg-New York, 1971.

Technion

Deparment of Mathematics

Haifa 32000

Israel

Received 27 August 1982 